\title{
Factors Influencing Participation in Biospecimen Research among Parents of Youth with Mental Health Conditions
}

\author{
Ashli A. Owen-Smith ${ }^{a, b}$ Musu M. Sesay ${ }^{b}$ Frances L. Lynch ${ }^{c}$ Maria Massolo $^{d}$ \\ ${\text { Hilda } \text { Cerros }^{d} \text { Lisa A. Croen }}^{d}$

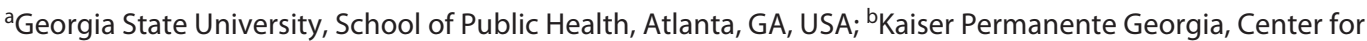 \\ Research and Evaluation, Atlanta, GA, USA; 'Kaiser Permanente Northwest, Center for Health Research, Portland, \\ OR, USA; ${ }^{d}$ Kaiser Permanente Northern California, Division of Research, Oakland, CA, USA
}

\section{Keywords}

Biospecimen · Biobanking · Mental health · Children ·

Research participation

\begin{abstract}
Introduction: Biospecimens are tools that have the potential to improve early identification and treatment for autism spectrum disorders (ASD) and bipolar disorders (BPD). Unfortunately, most biobanks lack racial/ethnic diversity. One challenge to including a diverse sample of youth is recruiting and engaging families. Objective: We sought to better understand facilitators and barriers to participation in biospecimen research among a diverse group of parents of youth with ASD and BPD. Methods: The current study involved 3 Mental Health Research Network sites. At each site, parents participated in an interview that explored attitudes and beliefs about genetic research. Interviews were audio-recorded, and audio files were transcribed and coded using content analysis. Results: A total of 58 interviews were conducted. Four challenges emerged: (1) contacting and engaging potential research participants, (2) motivating potential participants to read recruitment and consent materials, (3) motivating participation in research, in general, and (4) motivat-
\end{abstract}

ing participation in research involving biospecimen donation, specifically. Conclusions: Participants were eager to participate as long as the research process involved trust, clarity, and flexibility. Future research involving youth with mental health conditions would benefit from implementing multimodal strategies for recruitment and data collection and sharing knowledge gained by the research with study participants.

(c) 2020 S. Karger AG, Basel

\section{Introduction}

Evidence suggests that $13-20 \%$ of youth living in the USA (up to 1 out of 5 youth) experience a mental illness in a given year [1]. Two of the most impairing mental health conditions affecting youth and adolescents in this country are autism spectrum disorders (ASD) and bipolar disorder (BPD), and both are increasing in prevalence. ASD, a developmental disability, can cause significant impairment in behavioral, educational, interpersonal, and social skills [2]; BPD is a mental illness that causes unusual shifts in mood, energy, activity levels, concentration, and difficulty in functioning in day-to-day tasks [3].

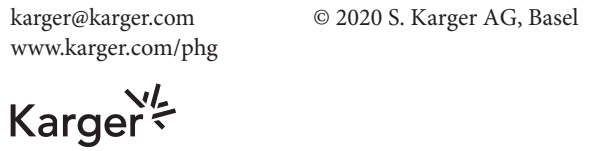

Ashli A. Owen-Smith

Urban Life Building, School of Public Health, Georgia State University 140 Decatur Street, Suite 434

Atlanta, GA 30303 (USA)

aowensmith@gsu.edu 
The prevalence of ASD has rapidly increased over the last 54 years from 1 in 2,000 [4] to 1 in 54 today [5]. The lifetime prevalence of BPD is $2.9 \%$ [6], and despite the lack of consensus criteria for diagnosis, the rate of diagnoses of BPD in youth has been increasing rapidly [7]. Both conditions are strongly familial, with a sibling recurrence risk of approximately $20 \%$ for ASD [8] and a 7-fold increased risk of bipolar I disorder in the relatives of youth with bipolar I disorder [9]. Both ASD and BPD have significant economic and social consequences for the individual, families, and society as a whole [10-13]. Parents who are the primary caregivers of the affected child often report significant mental and emotional strain (e.g., high levels of stress, worry, and sadness/grief), health-related issues (e.g., decreased energy and declined self-care), social issues (e.g., perceived stigma), work-related problems (e.g., inadequate childcare, distractions during the workday, and absences from work), and family issues (e.g., conflicted sibling relationships and marital discord) [14].

Diagnosis and treatment of both ASD and BPD in youth are complex and often time consuming. Biospecimens are objective tools that have the potential to improve early identification and the testing of treatment effects for these illnesses. For example, the use of biomarkers could help identify homogeneous subgroups of patients and test treatments targeting a particular biomedical process $[15,16]$. Similarly, biomarkers could aid in the identification of a genetic fingerprint predicting individual response to a drug that could explain heterogeneity of treatment response [17].

Biospecimen collection and the genetic testing of those biospecimens is growing rapidly in the USA [18-22] and can facilitate discovery of new screening tests and treatments. Unfortunately, biospecimen collection from youth is less common, in part due to the additional ethical issues posed by this research. Youth have limited capacity to understand the purpose and implications of the research and provide informed consent $[23,24]$ and the need to re-consent youth at maturity poses significant practical and ethical concerns. Biospecimen collection from youth with mental health conditions is even less common, as these individuals are members of 2 vulnerable population groups and, thus, additional human subject protections are required. Further, parents of youth with mental health conditions may be less likely to enroll their child in a biospecimen research study, as evidence suggests that parenting a child who needs constant supervision and behavioral interventions can make it difficult to participate in other activities, particularly those that involve expectations for certain types of behavioral compliance [25].
An additional limitation to current biospecimen collection is the lack of diversity in most biobanks [26, 27], with most samples including mainly non-Hispanic white participants [28]. Racial/ethnic minorities may be less likely to participate in biobanks because of limited knowledge about genetics, misperceptions of risk, and distrust of the medical system [29-31]. Lower participation rates among racial/ethnic minorities may also be a function of socioeconomic status, as evidence suggests that higher income individuals may have better access to opportunities to provide a biospecimen due to health insurance coverage, accessibility of testing, availability of health-related services if conditions are identified through biospecimen screening, and proximity to large research centers [32]. To address the significant disparities in health status and healthcare delivery that exists across racial/ethnic lines, it is imperative to increase the level of participation of racial/ethnic minorities in health-related research, including studies that use biospecimens [33].

A central challenge to including a more diverse sample of youth in biospecimen research is the ability to recruit and engage youth and their families in this type of research participation $[34,35]$. A variety of methods have been used for recruiting and consenting families to provide biospecimens (contact by mail, telephone, and/or Web). However, there is a paucity of research comparing the effectiveness of different methods, particularly their effectiveness for specific population groups (e.g., those with mental health conditions). In addition, little is known regarding the most acceptable methods to parents for recruiting, engaging, and consenting to participate in biospecimen research involving youth with mental health conditions. We sought to address these questions by conducting qualitative interviews with parents of youth with ASD and BPD who represented a diverse range of racial, ethnic, and socioeconomic groups. We sought to understand facilitators and barriers to participation in biospecimen research at both the engagement stage of research participation and the practical stage of specimen collection.

\section{Materials and Methods}

\section{Study Sites}

The current study involved 3 Mental Health Research Network (MHRN) Kaiser Permanente sites (Georgia [KPGA], Oregon/ Southwest Washington [KPNW], and Northern California [KPNC]). The MHRN is a consortium of research centers located within 13 large healthcare systems, many of which also have affiliated health insurance plans. These integrated healthcare systems are part of the larger Health Care Systems Research Network, which includes 17 US-based health system members. MHRN-par- 
ticipating health systems serve over 12.5 million individuals across 15 states with diverse populations.

\section{Sampling and Recruitment}

We identified parents from the MHRN Autism Registry Project [36], an autism research resource across multiple MHRN sites which included (1) electronic medical record data, (2) parent-reported information collected through a Web-based survey about utilization of and satisfaction with services both inside and outside of the health plan and willingness to donate biospecimens for future research, and (3) biospecimens, collected from both the youth with ASD and their biological parent(s). For the current study, we invited parents to participate from among those who participated in the MHRN Autism Registry Project Web-based survey and parents who were invited but did not participate in the survey; among those who participated in the survey, we selected parents who gave consent to donate a biospecimen for their child and parents who did not consent to child biospecimen donation so as to capture a range of attitudes and experiences with healthcare research and services. Our goal was to interview parents across racial/ethnic (black/African American, Latino, Asian, or white) and socioeconomic strata as defined by the education and insurance status (private or Medicaid). In addition, at the KPNC site only, parents of youth with a diagnosis of BPD were identified using the presence of ICD-9 codes (ICD-9 296.00-296.06, 296.40-296.46, 296.50296.56, 296.60-196.66, 296.7, 296.80, and 296.89) on at least 2 separate occasions [37] in the patient's electronic medical record and invited to participate. Parents were mailed an invitation package that included an introductory letter, consent form, and opt-out response card. Parents who did not return the opt-out response card within 10 days were contacted by phone.

\section{Data Collection}

At each site, parents participated in an individual, semi-structured, 30- to 60-min phone interview. The interview consisted of 8 main questions, each followed by additional sub-questions and probes. Areas explored included attitudes and beliefs about genetic research, the child's experience with biospecimen donation (concerns about pain and discomfort), and perceptions of trust and mistrust of scientific research. Barriers to and facilitators of participation in research particularly involving biospecimen donation for genetic research with emphasis on the consenting process were also explored. Parents were also asked several sociodemographic questions, including their highest level of education completed, race/ethnicity, marital status, and total household income.

KPNC participants were able to participate in their preferred language (English or Spanish), while KPGA and KPNW participants completed interviews in English only (being able to speak/ understand English was part of the eligibility criteria for these sites). Interviews were audio-recorded. Audio files were transcribed verbatim by a professional company that handles English and Spanish files, and identifiers were redacted from the transcripts. All transcripts were checked for accuracy at each site. Interviews at each site were conducted by staff trained in qualitative methods.

\section{Data Analysis}

Coders at all 3 study sites (one per site) were trained by a cultural anthropologist (M.M.). Each coder read interviews 3 times before coding them line by line. Each site double-coded 4 inter-
Table 1. Characteristics of interview participants $(n=58)$

\begin{tabular}{lc}
\hline Parent gender & 9 \\
$\quad$ Male & 49 \\
Female & \\
Parent race & 33 \\
$\quad$ White & 10 \\
Black/African American & 3 \\
Asian & 9 \\
Other & 3 \\
Prefer not to answer & \\
Parent ethnicity & 13 \\
$\quad$ Hispanic & 11 \\
Parent income level & 44 \\
$\quad$ <USD 40,000 & 3 \\
$\quad \geq U S D$ 40,000 & \\
Missing & 43 \\
Child's diagnosis & 15 \\
ASD & \\
BPD & 13.9 (range: 7-19) \\
Child's mean age, years & 15.6 (range: 9-18) \\
ASD & \\
BPD
\end{tabular}

ASD, autism spectrum disorders; BPD, bipolar disorders.

views with the 2 other sites to achieve inter-rater reliability. Coders held monthly meetings over 6 months that included training and practice, creation of the data dictionary, coding practice, and calibration by comparing coded text. All discrepancies were discussed until consensus was reached.

NVivo version 11 was used for coding interviews. Researchers experienced in qualitative analysis at the 3 sites collaborated in the development of the coding nodes (data dictionary) which were revised by consensus. Two members of the team (A.O.-S. and M.M.) performed content analysis working through an iterative inductive process in which themes emerged from the voices of the participants themselves, rather than a priori from theory [38].

\section{Results}

\section{Description of Participants}

A total of 58 semi-structured interviews were conducted (Table 1). Approximately 75\% $(n=43)$ were parents of a child with ASD and the remaining $(n=15)$ were parents of a child with BPD. Most participants were female $(n=$ 49); almost half ( $n=26)$ were racial/ethnic minorities and almost one-fourth $(n=11)$ were individuals of lower socioeconomic status. The average age of the child at the time of the interview was 13.9 years among youth with ASD (range: $7-19$ years) and 15.6 years among youth with BPD (range: $9-18$ years) (Table 1 ). 


\section{Themes}

Several clear themes emerged from the analysis of the interview data. Below we discuss these themes in the context of 4 research-related challenges: (1) contacting and engaging potential research participants, (2) motivating potential participants to read recruitment and consent materials, (3) motivating participation in research and in general, and (4) motivating participation in research involving biospecimen donation, specifically.

\section{Challenge 1: Contacting and Engaging Potential}

Research Participants

There was no consensus on the best way to approach potential participants. When parents were asked how they prefer to be contacted for research studies, most preferred mailed letters or email because phone calls were seen as intrusive. Some parents felt they were not always available to take a call, often screened calls and/or indicated they would feel put on the spot on the telephone. One participant noted:

I think email is good because sometimes when someone calls you on the phone, you're not sure yet. And then, sometimes you can feel kind of on the spot. Like, "Oh, my gosh. Do I want to do it or not?" And then, you might kind of just like make a rash choice and then maybe change your mind later versus like an email you can think about it and then decide whether... you want to do it or not.

However, some parents preferred phone calls because they worried they would misplace or forget about mailed letters or they would not read emails unless they recognized the sender. Also, there was the perception by some that email was not a trustworthy form of communication and also not "legitimate" or "official." For example, one participant said:

We have so much spam and so much deception in e-mails, I don't personally trust it. I don't know what to trust now.

When asked about what factors might deter them from opening a recruitment letter or email, many parents indicated that they would not open materials from an organization or a return address that was unfamiliar to them or if the envelope looked like "junk mail." One participant commented:

If it looks like junk mail and it's just from ... somebody's name who's not going to be recognizable as, like, let's say a legitimate medical facility or person, I'm just going to throw it in the trash. I don't read mail that, you know, I don't have time to read all my mail. So, if it looks like junk mail or somebody that I really can't identify right off the bat, I just throw it away.
Another parent was very explicit about opening mail or reading emails if they came with the logo of the medical facility where they receive care:

Yes, absolutely if it has the [name of medical facility] on it, because I know it's ... going to affect either my son or myself, so that will be ... more likely that I would read that email. Sometimes, they send me junk, you know ... and I don't pay attention to that. But if you have the [name of medical facility] logo, then I know that it's something medical.

Some parents also specifically indicated that communication addressed to their child or "To the parents of [name of child]" would prompt them to read the materials. Finally, many parents of autistic youth noted that they would be more likely to open envelopes/emails that specifically had the word "autism" on the outside/in the subject heading:

I think putting in "autism research study." I know that might go into privacy or something, but I would look at that. Your help is needed with autism. If it has the word, "autism," I'm going to open it.

By contrast, the parents of youth with BPD did not indicate that they would be any more or less likely to open recruitment materials if they included "bipolar disorder."

\section{Challenge 2: Motivating Individuals to Read}

Recruitment and Consent Materials

Parents were asked about what aspects of recruitment and consent materials (e.g., aesthetics, formatting, and length) would make them more or less likely to read the materials. Many parents preferred brief recruitment materials formatted in bullets or in "Q \& A" style. One participant summed it up as follows:

I don't really care for long [letters], because you really want to get what's the point. What is it? So, I can kind of see for a little bit of ... question and answer and graphs, rather than kind of ... a long article. Quick questions, quick answers.

Many parents wanted materials that included pictures/other visual elements; these preferences were particularly common among slack/African-American and lower SES participants. For example, 1 parent said:

Maybe pictures with, I think, bullet points of what's important. Yeah ... something with maybe either some gloss or a picture would probably be a little more impactful as far as like picking it up and maybe hanging onto it.

In terms of questions parents would want answered by the recruitment materials, the most common responses included information about what participation would entail, where the research would take place, what time commitment/duration of participation would be required, whether there would be an incentive, how the data would 
be used and protected, and whether the data would be shared with participants when the study was complete. Participants also highlighted how important it was that they understand from these materials exactly what researchers hoped to accomplish and who would benefit from the research. For example, as one parent said:

I would have to have the information on what the research is for, what's going to happen - what the effects they're hoping for are going to be of the research.

Many parents reported not reading the consent form "word for word" and said they typically skim the document if it was more than 2-3 pages long; most preferred a 1 - to 2-page document. Some parents complained that consent forms often contained language that was difficult to understand. This complaint was commonly reported by parents of youth with ASD as well as BPD and across all race/ethnic and SES groups:

When you find like, you know, more than 1 page - that's what puts you off also ... if it is ... very long that means ... "What am I consenting for?" You don't know; you don't want to sign it unless you know it and, you don't want to read it ...? You don't have time. It's not simple language sometimes, and it just needs to be plain and to the point, and as short as possible, with the information on it with enough information. I guess, enough information to satisfy the law.

\section{Challenge 3: Motivating Participation in Research}

When parents were asked about factors that influenced their decision on whether to participate in research, inevitably barriers came up. Given Tuskegee [39] and other abuses experienced by their communities in the name of science, one hurdle reported most commonly by black/African-American participants was the distrust they feel toward research. In the words of one participant:

It's fine, as long as [there is] no kind of nefarious intent. We're very suspicious of medical research because of the history behind it.

Distrust was also expressed by white, non-Hispanic parents, but their suspicion was directed more commonly toward the possible greed of pharmaceutical companies and their hidden agenda in some research studies. A parent noted:

I think more so who's funding it. I would be kind of - a bit hesitant if it was a pharmaceutical company that was doing. I just, you know, I mean, if they find some breakthrough, are they going to make the medication be just super expensive or is it going to really benefit and help people or is it going to help their bottom line?

Although many parents mentioned issues related to privacy and confidentiality of protected health information (PHI) as potential concerns about research participa- tion, these concerns were predominantly expressed by parents of youth with BPD and not as often by parents of youth with ASD. Several parents noted:

I just don't like our government so I don't think I want them to learn any of our business. I wouldn't want my or my child's name attached to anything. I don't want anything to sort of be used against her.

There were also practical concerns that influenced whether a family would join a research study. Time and related logistical issues were paramount for most parents. They consistently wanted to know how much time participation would take, whether they would have to travel for a research appointment, how long it would take for them to travel to the research site, and how often they would have to go to research appointments. The parent below emphasized convenience of participation:

A big part of it is the time ... how long does it take. Is it something we - like you said, can we do at home, and something we got to travel somewhere to go do. The convenience. Convenience for everybody involved.

Many parents told us that altruism rather than incentives was the main motivator for participation, but if studies provided incentives, they would welcome them. In general, interviewees favored gift cards, especially those they could use anywhere (e.g., Visa card rather than a store-specific card) and/or if they received a gift card or gifts, especially for their child. One parent liked incentives, but spoke for other parents in stating that helping others was the driving force for joining studies:

Compensation doesn't matter, anyway, to us as a community. I mean, yeah, they're nice. Trust me, they're nice. But that's not the heart of it for us. If it's going to help somebody, if it's going to help my son eventually...

For this mother, a gift card from a study provides relief from economic burden while allowing her to do something special for her child:

I like it because ... if I get the gift card, it's going to be for him. So, when I get stuff like that ... I try to give it back to him, try to give him incentives for him to do stuff, and I tell him I'm going to take him there, and I know it's not going to come out of my pocket. Because I'm a single mom. So, if there's an incentive, I - it makes difference because at least I know I can give - I can buy him something that I can't give him out of my pocket.

In addition to incentives, covering the cost of travel was seen as important if the study was asking parents to travel as part of participation. This was particularly salient for parents of lower SES. Several parents did note that a healthcare evaluation or other healthcare services would be a valuable incentive for participation, and this was expressed by both parents of youth with ASD and 
BPD. While parents of youth with ASD tended to favor assessment and diagnostic evaluations, for some parents of BPD youth, skill building classes and resources would be effective motivators. A parent of a youth with ASD who participated in research received a valuable evaluation for the child:

The biggest incentive that I had on one [study], it was awesome, but [name of organization] did a full psychological evaluation on my son for free. So, getting that kind of information is very helpful, because insurance doesn't cover, you know, a neuropsych [sic!], and that's expensive.

For a parent of a child with BPD, behavioral therapy would be a desirable part of a study:

If there was, say, follow-up treatment and support offered. That would be a huge incentive for me. So, you know, with bipolar, if it would be part of, say, you know, research that included - I don't know - a group skill-building class that we got to attend, or a parent workshop, or, you know, a consultation with an expert, or something like that, that would be a really good incentive.

However, many parents felt that they would likely participate in order to help other families, regardless of any incentive. Wanting to help others was a strong motivating factor for many parents, and this attitude was commonly reported across both parents of youth with ASD and BPD as well as across all race/ethnic and SES groups. The possibility of advancing medical knowledge was a sufficient motivator for this participant:

Honestly, if I felt that the research was truly worthwhile, and could truly make some sort of medical breakthrough or medical gain, then incentives would not matter. You know. It'd just be - it's just the right thing to do. And if it's the right thing to do, it's the right thing to do whether you are compensated in any way or not.

Another factor that many parents indicated would increase their likelihood of participating was receiving study-related results from the researchers. This would make some individuals more likely to make accommodations to participate, such as traveling greater distances and willing to receive smaller financial incentives. Several participants expressed feeling frustrated with prior participation experiences because they were not contacted by the researchers after the conclusion of the study with any study-related findings. Several parents noted specific reasons for wanting results:

I think if it's anything that concerns my child, I would definitely want to see the results. Because personally, I do anything I can to learn everything I can about my child ... To be able to see the results would give - I think, would give parents an opportunity to get to know what's going on with their child better. And that's definitely something I would want.

Biospecimen Research Participation
Though some parents expressed concern about the sharing of their data with other researchers (e.g., due to requirements to share data with the government for NIHfunded studies), most felt that this would not influence their decision to participate as long as there were adequate privacy/confidentiality protections in place. Providing assurance that their privacy would be protected is enough for this parent:

The main [thing] I would look for is, you know, are they going to provide personal information about my child? You know, I wouldn't want that. But, as long as I can see that it's going to be anonymous, then you know, I don't mind having my information shared. So, I'm not - I guess, I'm not all that worried.

For the participant below, sharing study-related data was seen as a facilitator to research participation:

It's important that they share the information so everyone, you know, can learn from each other what they found out and help try to figure this stuff out quicker, so everybody can teach each other.

\section{Challenge 4: Motivating Participation in Research}

Involving Biospecimen Donation

Time and logistical constraints were perceived as common barriers to participating in studies involving biospecimen donation. Many parents expressed that they would be more inclined to participate if biospecimen collection was done either at home (e.g., saliva samples that could be returned by mail) or collected at the same time as a medical office visit that they already had scheduled (so they were not making a special trip to the laboratory, which was not seen as realistic). These attitudes were common by both parents of youth with ASD and BPD as well as across all racial/ethnic and SES groups. Several parents echoed the following opinion:

I would prefer to do it at home. I mean, if I have to schedule it, that's going to take some planning, you know, and cause more anxiety for me, trying to balance schedules. So, I'm more likely to do it sooner if I do it at home.

Another issue that would influence participation concerned the specific type of biospecimen being collected: blood donation for some was challenging because it was traumatizing and or painful for their youth (although this was more often a concern for parents of youth with ASD and less so for parents of youth with BPD) and warranted a clinic visit which introduced time and logistical constraints. Other parents, like the one below, felt that blood donation was acceptable as long as they felt it was worthwhile. Some parents, particularly those with youth with ASD, echoed this participant's statement about the importance of preparing the child for the event:

Public Health Genomics 2020;23:122-132 
The first time we considered it, you know, I wondered if, you know, he would get scared or upset. And - but then, you know, I kind of thought about it being for, like, the greater good. So, when I made the decision, I sat him down and told him what was going to happen. And-you know, I'm, like, "A, we're going to go in. They're going to take some blood. They're going to swab your mouth. And this is why." And, you know - because he has full knowledge that he has this issue. And I'm, like, "And so, it's to help the-maybe, help future generations, you know. So, it's important to try and do what we can. So, that's why we keep doing it, you know."

\section{Discussion}

The semi-structured qualitative interviews we conducted with parents of youth with ASD or BPD provides new insights regarding barriers and facilitators to participation in research studies, particularly those involving biospecimen donation. Our findings underscore the value of taking a multimodal approach (e.g., email, mailed letters, and phone calls) to ensure reaching and engaging a larger and more diverse population. The perceived legitimacy of recruitment materials was important to many parents, who consistently indicated that they would be more likely to participate if the email/letter was from a recognizable and trusted source. Researchers often spend energy and resources creating study logos, but correspondence with study logos that are unfamiliar to potential participants may be ignored. Thus, using the logo from the individual's trusted healthcare/health insurance provider, if possible, may be more successful at engaging potential participants. Interestingly, many parents of youth with ASD indicated that they would be more likely to open recruitment materials that included some mention of ASD upfront (on the envelope for mailed materials, in the subject line on emails), whereas a mention of BPD in this fashion was not seen as important to parents of youth with BPD.

Not surprisingly, parents consistently reported that they would be more likely to read recruitment and informed consent materials that are clear and easy to read. These findings are consistent with prior research indicating that long and/or complex participant materials have a negative impact on recruitment, particularly when these documents are visually unappealing, confusing, or raise anxiety [40]. Researchers would be well advised to use shorter sentences, one-line/bulleted text, and images to convey key points. Reliable and valid readability tests, such as The Flesch-Kincaid Readability Scale [41], should be used when drafting participant materials. Partnering with patient stakeholders in the development of these ma- terials is also critical [42]. We acknowledge, however, that implementing these recommendations can be challenging, as there exists a profound tension between what parents want in terms of length and density of recruitment and consent forms and what is required by institutional review boards (IRBs). Informed consent necessitates that potential research participants read and understand the materials provided, but if they are too long or complex, informed consent may not occur. IRBs and researchers need to work together to ensure that adequate information about the study is shared in a way that maximizes the likelihood that study materials will be read and understood.

Consistent with prior research [43-45], trust (or lack thereof) was an important factor that served to either motivate or discourage research participation, respectively. The history of abuse by medical professionals was a source of distrust for some black/African-American participants, while concerns about the influence of pharmaceutical funding was a source of distrust for some white participants. Parents of youth with BPD more often expressed concerns about possible breaches of PHI privacy/ confidentiality than parents of youth with ASD, which may be the result of greater sociocultural stigma toward individuals with BPD than ASD [46]. Regardless of sociodemographic and clinical characteristics, distrust was reinforced when researchers failed to share study-related results with participants. "Helicopter" research - in which researchers literally or figuratively fly into study participants' communities, collect data, and leave, never to be heard from again by the community - erodes trust between the researcher and the study participant and invalidates the contribution made by that participant to the project and the larger scientific community [47]. While there are a host of legal, ethical, and pragmatic reasons for not sharing individual results with research participants [48], several recent biobanking efforts have incorporated patient and parental preferences into return-of-results decision-making $[49,50]$. Sharing a summary of study findings, written in lay terms, would bolster participant trust in the research enterprise. Additional strategies for enhancing trust between researchers and potential participants include involving community members in the design and implementation of research; for example, the use of lay outreach workers from the target population has been particularly effective in prior studies [33]. Working with community-based and/or advocacy organizations has also been an effective engagement strategy in prior research [33]. In our study, for example, several participants did mention Autism Speaks as a trusted source; 
thus, future research could specifically assess which organizations are perceived to be legitimate by the target population and prioritize working with these organizations throughout the research development, implementation, and dissemination process.

Some parents mentioned that the ability to access certain diagnostic services and/or treatments for their child was a strong incentive to participate in a study, which is consistent with reports from other research [51]. Parents of youth with ASD seemed to prioritize diagnostic and assessment-related services, while parents of youth with BPD seemed to favor access to skill-building classes and resources. Townsend and Cox have attempted to explain this specific motivation to participate in research as "therapeutic research participation," whereby individuals are cognizant of the fact that they are participating in research to gain access to services but understand the ways in which what they receive in the context of a study might be different from traditional health care (in contrast to the notion of "therapeutic misconception" in which individuals conflate research and treatment) [52]. However, even if participants are well informed about the risks and limits of what research can offer, the availability of diagnostic tests or services as part of research, particularly those which are expensive or inaccessible to some families, may create undue incentive structures for participation. This raises important ethical implications that warrant consideration, particularly given that families of youth with ASD often face significant economic barriers to accessing screening, diagnostic services, and treatments [53], and thus, for some, research participation may be one of the only ways less economically advantaged families can access cutting-edge care. Certainly, this underscores the need for a thorough but simple and clearly worded consent form that ensures potential participants are not conflating care and clinical research; however, the possibility of even a subtle coercion is worrisome. In addition to the need for more comprehensive state insurance mandates for ASD-related services (which has been shown to improve access to care [54]), there may be more immediate implications for ethical oversight in studies that provide services to participants, especially for families of youth with mental health conditions who may be particularly in need of those services.

One issue that emerged in the present research that has also been in prior studies $[44,45,55]$ is that logistical and financial factors can present significant barriers to participating in research. Many participants emphasized that participation needed to be "easy." These perceived facilitators to participation were particularly important for parents in this study, given that they were already overburdened with managing the personal, social, and economic challenges associated with caring for a child with special needs and, thus, reported having even less free time than other parents without these responsibilities. Therefore, designing recruitment and data collection procedures that are convenient (e.g., linking research visits to already-scheduled clinic visits), flexible (e.g., providing several options for study sites and days/times of the week.), and do not add any financial burden (e.g., incentives for participation should ideally include covering the cost of transportation, food, and childcare if needed) would enhance the research participation of diverse populations. It may also be important for researchers to consider implementing flexible data collection methods as well, whenever possible; for example, if participants are not able or willing to complete a survey online, alternatives such as completion by telephone or in-person could be offered instead. Indeed, this strategy may be especially critical when recruiting members of socially disadvantaged groups [56]. Incorporating home-based data collection (whereby individuals collect and then mail biospecimens to researchers) and/or home-based data collection and analysis (whereby individuals collect and analyze their own biospecimens) are other strategies that may increase research participation because of convenience, cost, and privacy, particularly for urine and saliva as these specimens are relatively easy to collect from all agegroups, including young children $[57,58]$.

Recent studies focused on attitudes and beliefs about participating in biospecimen research of diverse populations overwhelmingly suggest that participants report interest and willingness to participate in biobanking for altruistic purposes, particularly to benefit future generations [59-62]; this was certainly the case for the parents of youth with ASD and BPD. Also consistent with prior studies, our participants noted the importance of convenience and accessibility - and that they would be much more likely to donate a biospecimen if they could do so from home or during a routine healthcare encounter. However, parents - particularly those of youth with ASD - expressed concerns related to negotiating this experience with their youth, many of whom become distressed during saliva swabbing or blood draws; this may serve as a unique barrier to participation for these families. Results from a recent study on the feasibility of conducting biospecimen research with autistic youth confirm this, as over half of parents indicated that the blood draw requirement was their reason for nonparticipation [63]. One way to address this challenge may be for re- 
searchers to provide developmentally appropriate educational tools that parents can use to explain the procedures and prepare their youth in advance. For example, the Blood Draw Intervention Program toolkit includes a social story and practice materials to be used by parents with their autistic child, and its use has been associated with a 5 -fold increase in blood draw completion rates in this population [64]. Future research is needed to determine the effectiveness of these types of tools for youth with other mental health conditions such as BPD. Future research is also needed to explore the child's perceptions of/experiences with biospecimen donation, as much of the research on this topic has only focused on parental knowledge, attitudes, and beliefs.

\section{Limitations and Strengths}

The present study has several limitations. First, we did not have parental involvement during the design of the study or in the interpretation of study results; therefore, our participant recruitment and consent materials may not have been maximally effective, and our interview questions and thematic analysis may not have thoroughly described the nuances of parents' experiences. Second, though we attempted to recruit parents who both agreed and declined to participate in the MHRN Autism Registry Project, our study population was overrepresented by families who had participated in this prior study. Therefore, we may not have fully captured the attitudes and beliefs of nonparticipants who may have unique perspectives about barriers and facilitators to research engagement. Third, all interviews were conducted over the telephone, and thus, we may have missed respondents' informal, nonverbal communication [65]. Fourth, our sample included parents of youth with ages than ranged from 7 to 19 years old; future research could examine whether parental knowledge, attitudes, preferences, and experiences regarding biospecimen research participation might differ for parents of younger children. Finally, all participants were members of integrated healthcare systems, and thus, caution is urged in generalizing the findings to uninsured populations. However, it is worth noting that members do include those receiving government-sponsored insurance (e.g., Medicaid) and our sample was diverse with respect to socioeconomic status.

This study's strengths include a relatively large sample for a qualitative study and a large, geographically and racially/ethnically diverse study population which was focused on two different mental health conditions specifically in youth. To our knowledge, this is the only study of its kind with all three features.

\section{Conclusions}

In spite of the profound caregiver burden experienced by many parents of youth with serious mental health-related issues, participants in the present study were eager to participate in research, in general, and in biospecimen research, in particular, as long as the research process involved trust, clarity, and flexibility. Future research involving similar populations would benefit from designing and implementing multimodal strategies for recruitment and data collection and prioritizing the sharing of knowledge gained by the research with study participants at the end of the study.

\section{Statement of Ethics}

Participants provided verbal informed consent and the study protocol was approved by the institute's committee on human research. The Institutional Review Boards at each site approved data use for this project. Verbal consent to participate in the study was obtained by phone and audio-recorded.

\section{Conflict of Interest Statement}

The authors have no conflicts of interest to declare.

\section{Funding Sources}

Research reported in this publication was supported by the $\mathrm{Na}$ tional Institute of Mental Health of the National Institutes of Health under award number U19MH092201 (PI: Simon). The content is solely the responsibility of the authors and does not necessarily represent the official views of the National Institutes of Health. The data that support the findings of this study are available from the corresponding author upon reasonable request.

\section{Author Contributions}

All authors contributed to the implementation of the study protocol/data collection, analysis, and drafting the manuscript. All authors approved the final version of the manuscript.

\footnotetext{
References

1 Centers for Disease Control and Prevention What are childhood mental disorders? 2020.

2 Centers for Disease Control and Prevention. What is autism spectrum disorder? 2020.

3 National Institutes of Mental Health. Bipolar disorder. 2020.

4 Lotter V. Epidemiology of autistic conditions in young children. Social Psychiatry Psychiatr Epidemiol. 1966;1:124-35.
} 
5 Baio J, Wiggins L, Christensen DL, Maenner MJ, Daniels J, Warren Z, et al. Prevalence of autism spectrum disorder among children aged 8 years: autism and developmental disabilities monitoring network, 11 sites, United States, 2016. MMWR Surveill Summ. 2018 Apr 27;67(6):1-23.

6 Merikangas KR, He JP, Burstein M, Swanson SA, Avenevoli S, Cui L, et al. Lifetime prevalence of mental disorders in U.S. adolescents: results from the National Comorbidity Survey Replication-Adolescent Supplement (NCS-A). J Am Acad Child Adolesc Psychiatry. 2010;49:980-9.

7 Papolos DF, Bronsteen A. Bipolar disorder in children: assessment in general pediatric practice. Curr Opin Pediatr. 2013;25:419-26.

8 Ozonoff S, Young GS, Carter A, Messinger D, Yirmiya N, Zwaigenbaum L, et al. Recurrence risk for autism spectrum disorders: a baby siblings research consortium study. Pediatrics. 2011;128:e488-495.

9 Wozniak J, Faraone SV, Martelon M, McKillop HN, Biederman J. Further evidence for robust familiality of pediatric bipolar I disorder: results from a very large controlled family study of pediatric bipolar I disorder and a meta-analysis. J Clin Psychiatry. 2012;73: 1328-34.

10 Guevara JP, Mandell DS, Rostain AL, Zhao H, Hadley TR. National estimates of health services expenditures for children with behavioral disorders: an analysis of the medical expenditure panel survey. Pediatrics. 2003;112: e440.

11 Stimmel GL. Economic grand rounds: the economic burden of bipolar disorder. Psychiatr Serv. 2004;55:117-8.

12 Ganz ML. The lifetime distribution of the incremental societal costs of autism. Arch Pediatr Adolesc Med. 2007;161:343-9.

13 Cidav Z, Marcus SC, Mandell DS. Implications of childhood autism for parental employment and earnings. Pediatrics. 2012;129: $617-23$.

14 Mendenhall AN, Mount K. Parents of children with mental illness: exploring the caregiver experience and caregiver-focused interventions. Families Soc J Contemp Social Serv. 2011;92:183-90.

15 Bent S, Hendren RL. Improving the prediction of response to therapy in autism. Neurotherapeutics. 2010;7:232-40.

16 Hendren RL. Autism: biomedical complementary treatment approaches. Child Adolesc Psychiatr Clin N Am. 2013;22:443-56.

17 Burt T, Dhillon S. Pharmacogenomics in early-phase clinical development. Pharmacogenomics. 2013;14:1085-97.

18 McCarty CA, Chapman-Stone D, Derfus T, Giampietro PF, Fost N, Marshfield Clinic PMRP Community Advisory Group. Community consultation and communication for a population-based DNA biobank: the Marshfield Clinic Personalized Medicine Research Project. Am J Med Genet A. 2008; 146A:3026-3033.
19 Ormond KE, Cirino AL, Helenowski IB, Chisholm RL, Wolf WA. Assessing the understanding of biobank participants. Am J Med Genet A. 2009;149:188-98.

20 Roden DM, Pulley JM, Basford MA, Bernard GR, Clayton EW, Balser JR, et al. Development of a large-scale de-identified DNA biobank to enable personalized medicine. Clin Pharmacol Ther. 2008;84:362-9.

21 Vaught J. Biobanking and beyond: the importance of scientific collections. Biopreserv Biobank. 2019;17:1.

22 Yang Q, Khoury MJ, Botto L, Friedman JM, Flanders WD. Improving the prediction of complex diseases by testing for multiple disease-susceptibility genes. Am J Hum Genet. 2003;72:636-49.

23 Beskow LM, Lin L, Dombeck CB, Gao E, Weinfurt KP. Improving biobank consent comprehension: a national randomized survey to assess the effect of a simplified form and review/retest intervention. Genet Med. 2017; 19:505-12.

24 Samuel J, Knoppers BM, Avard D. Paediatric biobanks: what makes them so unique? J Paediatr Child Health. 2012;48:E1-3.

25 Luther EH, Canham DL, Young Cureton V. Coping and social support for parents of children with autism. J Sch Nurs. 2005;21:40-7.

26 Haga SB. Impact of limited population diversity of genome-wide association studies. Genet Med. 2010;12:81-4.

27 Kim P, Milliken EL. Minority participation in biobanks: an essential key to progress. Methods Mol Biol. 2019;1897:43-50.

28 Anckarsäter H, Lundström S, Kollberg L, Kerekes N, Palm C, Carlström E, et al. The child and adolescent twin study in Sweden (CATSS). Twin Res Hum Genet. 2011;14: 495-508.

29 Dash C, Wallington SF, Muthra S, Dodson E, Mandelblatt J, Adams-Campbell LL. Disparities in knowledge and willingness to donate research biospecimens: a mixed-methods study in an underserved urban community. J Community Genet. 2014;5:329-36.

30 Hagiwara N, Berry-Bobovski L, Francis C, Ramsey L, Chapman RA, Albrecht TL. Unexpected findings in the exploration of African American underrepresentation in biospecimen collection and biobanks. J Cancer Educ. 2014;29:580-7.

31 Heredia NI, Krasny S, Strong LL, Von Hatten L, Nguyen L, Reininger BM, et al. Community perceptions of biobanking participation: a qualitative study among Mexican-Americans in three Texas cities. Public Health Genomics. 2017;20:46-57.

32 Lakes KD, Vaughan E, Jones M, Burke W, Baker D, Swanson JM. Diverse perceptions of the informed consent process: implications for the recruitment and participation of diverse communities in the National Children's Study. Am J Community Psychol. 2012;49: 215-32.
33 Yancey AK, Ortega AN, Kumanyika SK. Effective recruitment and retention of minority research participants. Annu Rev Public Health. 2006;27:1-28.

34 Antommaria AHM, Brothers KB, Myers JA, Feygin YB, Aufox SA, Brilliant MH, et al. Parents' attitudes toward consent and data sharing in biobanks: a multisite experimental survey. AJOB Empir Bioeth. 2018;9:128-42.

35 Wagner KE, McCormick JB, Barns S, Carney M, Middleton FA, Hicks SD. Parent perspectives towards genetic and epigenetic testing for autism spectrum disorder. J Autism Dev Disord. 2019.

36 Becerra TA, Massolo ML, Yau VM, OwenSmith AA, Lynch FL, Crawford PM, et al. A survey of parents with children on the autism spectrum: experience with services and treatments. Perm J. 2017;21:16-19.

37 Coleman KJ, Lutsky MA, Yau V, Qian Y, Pomichowski ME, Crawford PM, et al. Validation of autism spectrum disorder diagnoses in large healthcare systems with electronic medical records. J Autism Dev Disord. 2015;45: 1989-96.

38 Crowe $\mathrm{M}$, Inder $\mathrm{M}$, Porter R. Conducting qualitative research in mental health: thematic and content analyses. Aust N Z J Psychiatry. 2015;49:616-23.

39 Shavers VL, Lynch CF, Burmeister LF. Knowledge of the Tuskegee study and its impact on the willingness to participate in medical research studies. J Natl Med Assoc. 2000; 92:563-72.

40 Man MS, Rick J, Bower P. Improving recruitment to a study of telehealth management for long-term conditions in primary care: two embedded, randomised controlled trials of optimised patient information materials. Trials. 2015;16:309.

41 Kincaid JP, Fishburne RP, Rogers RL, Chissom BS. Derivation of new readability formulas (Automated Readability Index, Fog Count, and Flesch Reading Ease Formula) for Navy enlisted personnel. Millington: Institute for Simulation and Training; 1975. p. 8-75.

42 Mitchell D, Geissler J, Parry-Jones A, Keulen $\mathrm{H}$, Schmitt DC, Vavassori R, et al. Biobanking from the patient perspective. Res Involv Engagem. 2015;1:4.

43 Gamble VN. A legacy of distrust: African Americans and medical research. Am J Prev Med. 1993;9:35-8.

44 Martinez P, Cummings C, Karriker-Jaffe KJ Chartier KG. Learning from Latino voices: focus groups' insights on participation in genetic research. Am J Addict. 2017;26:477-85.

45 Woodall A, Morgan C, Sloan C, Howard L. Barriers to participation in mental health research: are there specific gender, ethnicity and age related barriers? BMC Psychiatry. 2010; 10:103.

46 Durand-Zaleski I, Scott J, Rouillon F, Leboyer M. A first national survey of knowledge, attitudes and behaviours towards schizophrenia, bipolar disorders and autism in France. BMC Psychiatry. 2012;12:128. 
47 Ferreira MP, Gendron F. Community-based participatory research with traditional and indigenous communities of the Americas: historical context and future directions. Int $\mathrm{J}$ Critical Pedagogy. 2011;3:153-68.

48 Fernandez CV, Kodish E, Weijer C. Informing study participants of research results: an ethical imperative. IRB. 2003;25:12-9.

49 Christensen KD, Savage SK, Huntington NL, Weitzman ER, Ziniel SI, Bacon PL, et al. Preferences for the return of individual results from research on pediatric biobank samples. J Empir Res Hum Res Ethics. 2017;12:97-106.

50 Holm IA, Savage SK, Green RC, Juengst E, McGuire A, Kornetsky S, et al. Guidelines for return of research results from pediatric genomic studies: deliberations of the Boston Children's Hospital Gene Partnership Informed Cohort Oversight Board. Genet Med. 2014;16:547-52.

51 Tabor HK, Brazg T, Crouch J, Namey EE, Fullerton SM, Beskow LM, et al. Parent perspectives on pediatric genetic research and implications for genotype-driven research recruitment. J Empir Res Hum Res Ethics. 2011;6: 41-52.

52 Townsend A, Cox SM. Accessing health services through the back door: a qualitative interview study investigating reasons why people participate in health research in Canada. BMC Med Ethics. 2013;14:40.
53 Kogan MD, Strickland BB, Blumberg SJ, Singh GK, Perrin JM, van Dyck PC. A national profile of the health care experiences and family impact of autism spectrum disorder among children in the United States, 20052006. Pediatrics. 2008;122:e1149-58.

54 Barry CL, Epstein AJ, Marcus SC, KennedyHendricks A, Candon MK, Xie M, et al. Effects of state insurance mandates on health care use and spending for autism spectrum disorder. Health Aff. 2017;36:1754-61.

55 George S, Duran N, Norris K. A systematic review of barriers and facilitators to minority research participation among African Americans, Latinos, Asian Americans, and Pacific Islanders. Am J Public Health. 2014;104:e1631.

56 Bonevski B, Randell M, Paul C, Chapman K, Twyman L, Bryant J, et al. Reaching the hardto-reach: a systematic review of strategies for improving health and medical research with socially disadvantaged groups. BMC Med Res Methodol. 2014;14:42.

57 Rockett JC, Buck GM, Lynch CD, Perreault $\mathrm{SD}$. The value of home-based collection of biospecimens in reproductive epidemiology. Environ Health Perspect. 2004;112:94-104.

58 Granger DA, Johnson SB, Szanton SL, Out D, Schumann LL. Incorporating salivary biomarkers into nursing research: an overview and review of best practices. Biol Res Nurs. 2012;14:347-56.
59 Drake BF, Boyd D, Carter K, Gehlert S, Thompson VS. Barriers and strategies to participation in tissue research among AfricanAmerican men. J Cancer Educ. 2017;32:51-8.

60 Dang JH, Rodriguez EM, Luque JS, Erwin DO, Meade CD, Chen MS Jr. Engaging diverse populations about biospecimen donation for cancer research. J Community Genet. 2014;5:313-27.

61 McDonald JA, Vadaparampil S, Bowen D, Magwood G, Obeid JS, Jefferson M, et al. Intentions to donate to a biobank in a national sample of African Americans. Public Health Genomics. 2014;17:173-82.

62 Davis TC, Arnold CL, Mills G, Miele L. A qualitative study exploring barriers and facilitators of enrolling underrepresented populations in clinical trials and biobanking. Front Cell Dev Biol. 2019;7:74.

63 Sices L, Pawlowski K, Farfel L, Phillips D, Howe Y, Cochran DM, et al. Feasibility of conducting autism biomarker research in the clinical setting. J Dev Behav Pediatr. 2017;38: 483-92.

64 Davit CJ, Hundley RJ, Bacic JD, Hanson EM. A pilot study to improve venipuncture compliance in children and adolescents with autism spectrum disorders. J Dev Behav Pediatr. 2013;32:521-5

65 Creswell JW. Qualitative inquiry and research design: choosing among five traditions. Thousand Oaks, CA, Sage; 1998. 